\title{
Política de dividendos e volatilidade do preço das ações das empresas brasileiras de capital aberto
}

\author{
Dividend policy and volatility of the share price of brazilian publicly traded companies \\ Política de dividendos y volatilidad del precio de las acciones de empresas brasileñas con capital
}

abierto

Recebido: 23/03/2021 | Revisado: 03/04/2021 | Aceito: 06/04/2021 | Publicado: 17/04/2021

\author{
Arthur Antônio Silva Rosa \\ ORCID: https://orcid.org/0000-0003-4720-4791 \\ Universidade Federal de Uberlândia, Brasil \\ E-mail: arthursr@hotmail.com \\ Flávia Barbosa de Brito Araújo \\ ORCID: https://orcid.org/0000-0001-6137-379X \\ Universidade Federal de Uberlândia, Brasil \\ E-mail: flaviabbrito@yahoo.com.br \\ Pablo Rogers \\ ORCID: https://orcid.org/0000-0002-0093-3834 \\ Universidade Federal de Uberlândia, Brasil \\ E-mail: pablorogers@ufu.br
}

\begin{abstract}
Resumo
O objetivo deste estudo é analisar a relação entre a política de dividendos, a partir de duas das suas principais medidas - dividend yield e payout - e a volatilidade do preço das ações (VPA) no mercado brasileiro no período de 2010 a 2018: a amostra final foi composta por 357 empresas listada na B3 (Brasil, Bolsa e Balcão). Dois modelos de regressão com efeitos fixos a partir dos dados em painel foram utilizados como metodologia. Os resultados apontaram que as principais variáveis da política de dividendos influenciam de forma negativa a VPA, isto é, dado uma redução no dividend yield ou pagamento de dividendos, há um aumento na volatilidade do preço das ações das empresas brasileiras. Além disso, a alavancagem apresentou uma relação positiva com a VPA, enquanto o valor de mercado e o tamanho da companhia, apresentaram uma relação negativa com a variável dependente. Já a volatilidade do lucro, não apresentou significância estatística. A pesquisa contribui com a literatura por explorar um tema ainda sem consenso no cenário nacional, e trouxe implicações práticas para investidores e gestores, pois as informações sobre o dividend yield e o pagamento de dividendos podem auxiliar os mesmos a analisar e executar estratégias mais precisas sobre a análise financeira da empresa e na tomada de decisão sobre investimentos.
\end{abstract}

Palavras-chave: Volatilidade; Política de dividendos; Mercado de capitais brasileiro.

\begin{abstract}
The objective of this study is to analyze the relationship between the dividend policy, based on two of its main measures - dividend yield and payout - and the volatility of the share price (VPA) in the Brazilian market in the period from 2010 to 2018: the final sample consisted of 357 companies listed on B3 (Brazil, Bolsa and Balcão). Two multiple linear regression models were used as methodology, with effects fixed and estimated by panel data. The results showed that the main variables of the dividend policy negatively influence the VPA, that is, given a reduction in the dividend yield or payment of dividends, there is an increase in the volatility of the share price of Brazilian companies. In addition, leverage has a positive relationship with VPA, while market value and company size have a negative relationship with the dependent variable. Profit volatility, however, was not statistically significant. The research contributes to the literature by exploring a topic that still has no consensus on the national scene, and has practical implications for investors and managers, as information on dividend yield and dividend payment can help them to analyze and execute more accurate strategies on the company's financial analysis and investment decisionmaking.
\end{abstract}

Keywords: Volatility; Dividend policy; Brazilian capital market.

\section{Resumen}

El objetivo de este estudio es analizar la relación entre la política de dividendos, con base en dos de sus principales medidas - la rentabilidad y el pago de dividendos - y la volatilidad del precio de la acción (VPA) en el mercado brasileño en el período de 2010 a 2018: la muestra final estuvo formada por 357 empresas que cotizan en B3 (Brasil, Bolsa y Balcão). Se utilizaron como metodología dos modelos de regresión con efectos de efectos fijos a partir de los datos del panel. Los resultados mostraron que las principales variables de la política de dividendos influyen 
negativamente en el VPA, es decir, ante una reducción en la rentabilidad por dividendo o pago de dividendos, hay un aumento en la volatilidad del precio de las acciones de las empresas brasileñas. Además, el apalancamiento tiene una relación positiva con el VPA, mientras que el valor de mercado y el tamaño de la empresa tienen una relación negativa con la variable dependiente. La volatilidad de las ganancias, por otro lado, no fue estadísticamente significativa. La investigación contribuye a la literatura al explorar un tema que aún no tiene consenso en el escenario nacional, y tiene implicaciones prácticas para inversionistas y gerentes, ya que la información sobre rentabilidad de dividendos y pago de dividendos puede ayudarlos a analizar y ejecutar estrategias más precisas sobre el análisis financiero de la empresa y toma de decisiones de inversión.

Palabras clave: Volatilidad; Política de dividendos; Mercado de capitales brasileño.

\section{Introdução}

As pesquisas sobre as implicações da política de dividendos se tornaram ponto de partida a partir dos estudos de Lintner em 1956, que buscava determinar a política ideal de dividendos das empresas. A princípio, as finanças corporativas tratavam a política de dividendos como uma escolha de quanto e como seriam distribuídos os lucros da empresa para seus acionistas. Com o passar dos anos, o tema tem se tornado mais comum entre os assuntos corporativos financeiros e as evidências apresentaram mais que uma simples distribuição (Hussainey, Mgbame \& Chijoke-Mgbame, 2011). Assim, a política de dividendos não se restringe apenas em distribuir os lucros da empresa, mas em avaliar, projetar e aplicar com eficácia as estratégias ao longo do tempo.

A política de dividendos também apresenta implicações práticas e relevantes nas mudanças de preços das ações, pois é uma parte importante das estratégias de financiamento de longo prazo da empresa, uma vez que se refere a um meio de distribuição dos lucros através de pagamento aos seus acionistas/investidores, ora direcionando a empresa por meio de reinvestimento na empresa e recompra de ações (Hussainey, Mgbame \& Chijoke-Mgbame, 2011).

Desta forma, Miller e Modigliane (1961) ressaltaram que a política de dividendos em relação ao preço de uma ação é de suma importância para entender o mercado de capitais, dada a política de investimentos. Gordon (1963) destaca que a partir de um cenário baseado em incertezas, os investidores preferem receber dividendos o quanto antes. Fama et al. (1969), em seus estudos, ressaltaram que o mercado de ações pode ser considerado eficiente, de modo que os preços das ações se ajustam velozmente às novas informações.

Nesse sentido, vários estudiosos ao longo dos anos exploraram a relação entre a política de dividendos e a volatilidade do preço das ações a fim de detectar suas implicações, dentre eles Miller e Modigliani (1961), Gordon (1963), Elton e Gruber (1970), Pettit (1972), Black e Scholes (1974), Al-Malkawi (2007), Hussainey, Mgbame e Chijoke-Mgbame (2011), Hashemijoo, Ardekani e Younesi (2012), Hooi, Albaity e Ibrahimy (2015), Zainudin, Mahdzan e Yet (2018), Haque, Jahiruddin e Mishu (2019), entre outros. Todavia, os estudos apresentaram controvérsias, de maneira que algumas pesquisas são consistentes com a hipótese de que o aumento no pagamento de dividendos aumenta o preço das ações e outras com a hipótese de que o aumento no pagamento de dividendos reduz o preço das ações (Black \& Scholes, 1974).

Assim, várias teorias foram criadas para explicar a associação entre essas variáveis, o que divide opiniões. Dentre elas, a teoria sobre o efeito clientela, o efeito de informação ou sinalização e a teoria do pássaro na mão (Hussainey, Mgbame \& Chijoke-Mgbame, 2011).

Miller e Modigliani (1961) acreditavam na existência de preferências de investimentos: o efeito clientela diz respeito aos investidores que preferem ações de empresas que satisfaçam sua necessidade particular. Assim, as empresas podem atrair ou até mesmo afastar clientes com base em suas políticas de dividendos (Elton \& Gruber, 1970) de acordo com a taxa de pagamento específica de cada organização (Miller \& Modigliani, 1961).

Desta forma, argumenta-se que os investimentos muitas vezes são influenciados pelo comportamento dos investidores em relação à política de investimentos e aos tipos de riscos, pois eles buscarão obter informações disponíveis para a tomada de decisão e para reduzirem as possíveis incertezas do mercado. Assim, através da hipótese da sinalização, o mercado pode 
transmitir sinais sobre as organizações em relação ao desempenho, de modo que alterações nas políticas de dividendos influenciam as variações nos preços das ações provenientes da distribuição dos lucros (Loss \& Sarlo Neto, 2003). Pettit (1972) apoia a hipótese de que o mercado faz uso de anúncios de alterações nos pagamentos de dividendos para avaliação do valor de um título e que investidores atuam com base nas informações a serem anunciadas.

Do ponto de vista de Al-Malkawi (2007), em um mundo de incerteza e assimetria de informação, deve-se considerar que os dividendos são avaliados diferentemente dos lucros acumulados (ganhos de capital) e, assim, os investidores tendem a preferir pagamentos de dividendos/dinheiro imediato na mão - teoria do pássaro na mão. Entretanto, existem outros que preferem ter ganhos de capital para reduzir o impacto tributário, apesar de críticas acerca desses argumentos (Al-Malkawi, 2007; Hussainey, Mgbame \& Chijoke-Mgbame, 2011).

Esse tratamento diferenciado em relação aos dividendos se refere ao fato de receberem uma taxa tributária mais alta do que quando o ganho de capital é realizado na venda de uma ação, decorrendo de uma desvantagem fiscal no pagamento de dividendos. Porém, eles funcionam como um sinal dos fluxos de caixa esperado, sendo uma forma de sinalização da administração para enviar um sinal positivo sobre as perspectivas futuras do mercado e no preço das ações (Pettit, 1972; Hussainey, Mgbame \& Chijoke-Mgbame, 2011).

Apesar dos estudos acerca da política de dividendos e preço das ações se mostrarem contraditórios, diversas pesquisas relacionam a política de dividendos como forma de estratégia de investimento através da influência no preço das ações. Black e Sholes (1974) mencionam que uma redução no pagamento de dividendos de uma empresa pode causar uma queda temporária no preço das ações e argumentam que o melhor método para testar os efeitos das políticas de dividendos sobre os preços das ações é testar os efeitos do dividend yield nos retornos das ações. No entanto, Shiller (1981) e Leroy e Porter (1981) encontraram que a volatilidade do mercado de ações é muito maior do que poderia ser justificado por mudanças nos dividendos.

Em um estudo realizado na Austrália, Ball et. al. (1979) concluíram que existe uma relação positiva significativa entre a política de dividendos adotada pelas companhias e o retorno de suas ações. Segundo Baskin (1989), em um estudo realizado com empresas americanas, as práticas de distribuição de dividendos das empresas (utilizando como parâmetro o dividend yield) possuem uma relação positiva com o preço das ações das companhias. Ainda de acordo com a pesquisa, a volatilidade dos retornos e o tamanho da empresa afetam negativamente a volatilidade dos preços de suas ações, já a volatilidade dos retornos e o valor de sua dívida influenciam de forma positiva tal variável.

Hussainey, Mgbame e Chijoke-Mgbame (2011) utilizaram uma amostra de empresas listadas na Bolsa de Londres para analisar a política de dividendos corporativos, e enfatizaram que é um dos principais impulsionadores das mudanças no preço das ações no Reino Unido. Portanto, sinalizam uma relação positiva entre o rendimento de dividendos e a volatilidade no preço das ações (VPA), além de uma relação negativa entre a taxa de pagamento de dividendos e a VPA.

Hashemijoo, Ardekani e Younesi (2012), com foco nas empresas de produtos de consumo listadas na bolsa de valores da Malásia, durante 2005 a 2010, encontraram uma relação negativa entre a volatilidade do preço das ações com as duas medidas principais da política de dividendos: dividend yield e pagamento de dividendos.

Hooi, Albaity e Ibrahimy (2015), em uma pesquisa realizada com 319 empresas malasianas, encontraram uma relação negativa entre a política de dividendos relacionada ao pagamento de dividendos e a VPA. Em relação às variáveis de controle, os autores identificaram relações significantes e positivas entre VPA e dívidas de longo prazo, volatilidade de ganhos e uma relação negativa com o tamanho da empresa, enquanto o crescimento dos ativos não apresentou significância estatística.

Resultado semelhante foi encontrado por Zainudin, Mahdzan e Yet (2018). Ao utilizar empresas da Bolsa de Valores da Malásia concluíram que tanto o rendimento de dividendos quanto o pagamento de dividendos influenciam negativamente a VPA, efeito que é potencializado no período pós-crise. Também encontraram que a VPA possui uma relação negativa com o 
tamanho da companhia. Já a volatilidade dos lucros e a taxa de crescimento se relacionaram de forma positiva com a VPA, enquanto a alavancagem não apresentou indícios que afete tal variável.

Haque, Jahiruddin e Mishu (2019) também encontraram uma relação negativa entre as variáveis, tendo como amostra empresas manufatureiras listadas na Bolsa de Valores de Dhaka (DSE) de Bangladesh: as variáveis relacionadas a rendimento de dividendos e o tamanho da empresa apontaram uma relação inversa e significativa, por outro lado pagamento de dividendos apresentou associação positiva com a volatilidade do preço das ações.

Phan e Tran (2019) examinaram os impactos da política de dividendos na volatilidade do preço das ações no mercado vietnamita. Os resultados indicaram que a volatilidade dos preços não é influenciada pelo pagamento de dividendos, mas pelo rendimento de dividendos (dividend yield), uma vez que, empresas que oferecem rendimentos de dividendos mais elevados tendem a ser menos arriscadas em termos de oscilação de preço.

Em estudo similar, Khan, Jan e Khan (2020) realizaram um estudo de caso na Malásia para investigar a associação entre a política de dividendos e a volatilidade do preço das ações. Os resultados indicaram que um aumento no pagamento de dividendos reduzirá a volatilidade do preço das ações. De modo complementar, as estimativas indicaram que não existe significância entre o rendimento de dividendos e a volatilidade dos lucros sobre a volatilidade dos preços.

No Brasil, Santos Marques, Perpétuo e Rocha (2020) relataram que a política de dividendos tem sido bastante discutida, uma vez que, estudos tem apontado grande divergência sobre sua importância para os investidores e em relação ao valor de mercado. Alguns estudos como de Procianoy e Verdi (2003), Perobelli, Zanini e Santos (2009), De Pietro Neto, Decourt e Galli (2011), Martins e Famá (2012) e Silva e Machado (2015) buscaram entender a relação entre volatilidade dos preços e política de dividendos. Essas pesquisas proporcionaram vários métodos e uma diversidade de resultados, ou seja, ainda existe controvérsias sobre o tema.

Procianoy e Verdi (2003) pesquisaram sobre o efeito clientela no mercado brasileiro por meio do estudo de eventos, no período de 1989 a 1993 . Os resultados apontam que apenas 5\% das ações comportaram-se conforme o esperado no primeiro dia após o pagamento dos dividendos, e 47\% apresentaram que o preço da ação no primeiro dia ex-dividend foi maior do que na data em que a ação tinha direito a receber dividendos. Em 2009, Procianoy e Verdi produziram um estudo dando continuidade ao realizado em 2003 e identificaram resultados semelhantes.

Em contrapartida, Perobelli, Zanini e Santos (2009) promoveram uma pesquisa para discutir os impactos do pagamento de proventos sobre os preços das ações, na data ex direito, de empresas maduras e em expansão, considerando-se ainda o efeito da classe da ação (ordinária ou preferencial) sobre os resultados. Consideraram 61 ações, entre ordinárias e preferenciais, de 43 empresas, juntamente com informações sobre Dividendos e/ou Juros sobre Capital Próprio no período de 1996 a 2005. Os resultados revelaram que a estratégia de curto prazo de comprar ações na última data com direito ao recebimento dos proventos e vender a ação na primeira data ex direito e recebendo os dividendos, tanto para as ações ordinárias e preferenciais de empresas maduras quanto para as ações ordinárias de empresas em expansão, não tem sido vantajosa no mercado brasileiro.

As pesquisas de Medeiros e Van Doornik (2008) investigaram a relação empírica entre retorno acionário, volatilidade dos retornos e volume de negócios no mercado de ações brasileiro (Bovespa), no período de 2000 a 2005 . Os resultados indicaram uma correlação fraca, porém positivamente significativa, entre dividendos e as variações do volume de negócios, como também uma relação positiva entre volatilidade de retornos e volume de negócios. Os autores enfatizaram ainda que na literatura os estudos destacam que as flutuações de preço tendem a aumentar se houver um alto volume de negócios, especialmente em tempos de euforia nos mercados.

Referente aos anúncios à distribuição de proventos, De Pietro Neto, Decourt e Galli (2011) pesquisaram a teoria da sinalização, por meio das notícias transmitidas aos investidores, no período de 1998 a 2006. Os testes consideraram a presença 
de retornos anormais e acumulados, quando o mercado estava operando em níveis diferentes de volatilidade e de sentido de mercado. Considerando o sentido de mercado, quando o mercado operava em alta, os resultados encontrados em relação aos retornos anormais médios favoreceram o pagamento simultâneo de Juros Sobre o Capital Próprio e dividendos.

Martins e Famá (2012) produziram uma revisão da literatura sobre política de dividendos no Brasil e suas tendências, com foco nos estudos empíricos realizados no período de 1990 até 2010. Os autores destacam que as tendências dos estudos empíricos estão relacionadas à relevância da política de dividendos no mercado brasileiro; confirmação da existência de problemas de agência; efeito clientela; sinalização e influência dos tributos na definição da política de dividendos. Salientam, outrossim, que os estudos que apresentaram as variáveis de maior relevância relacionadas às determinantes da política de dividendos são o lucro líquido, o fluxo de caixa, o porte da empresa e o menor endividamento.

Silva e Machado (2015) investigaram a possível influência do dividend yield na VPA, e os resultados apontaram uma relação negativa entre variáveis. Também encontraram uma relação positiva com a dívida de longo prazo e negativa com o tamanho da empresa. A volatilidade dos lucros, por sua vez, não apresentou significância estatística.

Leite, Bambino e Hein (2017) analisaram a relação entre a política de dividendos e o desempenho econômicofinanceiro em empresas brasileiras e chilenas, no período de 2009 a 2013. Os autores destacaram que as decisões acerca da política de dividendos estão atreladas diretamente aos resultados das organizações, de modo a refletir no desempenho econômico-financeiro. Os resultados encontrados apresentaram influência negativa entre o conjunto de variáveis do grupo da política de dividendos no grupo de variáveis de desempenho econômico-financeiro.

Em um estudo bibliométrico sobre dividendos no Brasil no período de 2008 a 2017, De Sousa, Penedo e Pereira (2018) destacaram que as distribuições dos lucros são utilizadas para amenizar os conflitos de agência, o efeito clientela e a tributação, sendo fatores importantes para pagamento de dividendos. Os resultados apontaram que a política de dividendos se mostrou relevante nos últimos dez anos na área acadêmica brasileira, principalmente em finanças corporativas, assim como assunto para as principais revistas brasileiras.

Ao analisar a influência da flexibilidade financeira, exercida sob a forma de capacidade de endividamento, na distribuição de dividendos de 614 companhias brasileiras de capital aberto no período entre 2011 e 2016 , Rodrigues et al. (2019) destacaram que as empresas detentoras de capacidade de endividamento distribuem um volume maior de dividendos. Apontaram ainda que empresas maiores, mais lucrativas, mais líquidas e com maiores oportunidades de crescimento distribuem mais dividendos, enquanto empresas mais endividadas, com maior volume de investimentos e com resultados mais voláteis distribuem uma menor proporção de dividendos.

Em geral, os estudos apontam diversos resultados: algumas pesquisas indicam que o aumento no pagamento de dividendos aumenta o preço das ações; outras, que o aumento no pagamento de dividendos reduz o preço das ações (Black \& Scholes, 1974). Desta forma, a política de dividendos tem apresentado implicações práticas e relevantes em relação aos preços das ações, tornando-se uma ferramenta importante para as estratégias das empresas, além de um vasto potencial para pesquisas (Hussainey, Mgbame \& Chijoke-MGgbame, 2011).

A partir do contexto apresentado surgiu a seguinte situação-problema: Qual a influência da política de dividendos sobre a volatilidade do preço das ações das empresas brasileiras de capital aberto? Diante do exposto, este artigo tem por objetivo analisar a relação entre a política de dividendos das empresas e a volatilidade do preço das ações negociadas na B3 no período de 2010 a 2018.

Esta pesquisa apresenta-se relevante, pois auxiliará os investidores e gestores nas estratégias para a tomada de decisão de investimentos, de forma que possam analisar e prever os sinais da política de dividendo da companhia e que por vezes influenciam na VPA. Oferece ainda informações que dizem respeito a VPA, pois, quanto mais elevada for a volatilidade de uma ação, maior será a oscilação do preço e, assim, o risco de ganhar ou perder ao negociar determinada ação. 
Este artigo contribui com a literatura ao analisar dois modelos que relacionam a política de dividendos e à VPA. Os modelos propostos investigam separadamente as principais variáveis do estudo, de modo a verificar qual modelo apresenta implicações mais significativas em relação a VPA. Em síntese, os resultados deste estudo destacam que as principais variáveis da política de dividendos apresentaram uma relação negativa e significante com a volatilidade do preço das ações, isto é, dado uma redução no dividend yield e no pagamento de dividendos há um aumento na volatilidade do preço das ações das empresas brasileiras.

\section{Metodologia}

Essa pesquisa é do tipo descritiva e quantitativa e teve como objetivo deste estudo foi analisar a relação entre distribuição de dividendos e a volatilidade das ações. Para tanto, a amostra envolveu as empresas brasileiras que possuem o registro ativo ou cancelado na B3 no período de 2010 a 2018. Enfatiza-se que o recorte temporal para a análise a partir de 2010 tem por base a adoção dos novos padrões financeiros (International Financial Reporting Standards - IFRS).

A primeira fase da coleta de dados foi pautada em informações publicadas no banco de dados do software Economatica ${ }^{\circledR}$ e envolveu uma amostra de 632 empresas entre ativas e canceladas, com exclusão das empresas financeiras e companhias de seguros, devido às suas peculiaridades quanto à estrutura de capital. Posteriormente, foram excluídas as empresas que não apresentaram informações financeiras para a análise, com destaque para aquelas com valores menores que 3 anos consecutivos para o período analisado da pesquisa. Assim, a amostra finalizou com 357 empresas.

Optou-se por não se distinguir a distribuição de dividendos dos juros sobre o capital próprio (Forti, Peixoto \& Alves, 2015), ou seja, foi considerada a distribuição de recursos como um todo. E para se avaliar as implicações da política de dividendos sobre a volatilidade das ações, o presente artigo aplicou técnicas de regressão com dados em painel. Conforme indica Baum (2006), a análise utilizou no modelo econométrico testes como Dftis, que a partir da análise identificaram a presença de outliers multivariados na amostra: considerando todos os modelos em média excluímos 35 observações.

Quanto a detectar a presença de multicolinearidade, foi realizado o teste Variance Inflation Factor (VIF), que apresentou em média 1,37, indicando atender aos pressupostos recomendados (VIF < 5), conforme Fávero et al. (2009). Procedeu-se então aos testes de diagnóstico de Wooldridge, Wald, Chow, Breusch-Pagan LM e Hausmman. Nesse sentido, os testes de Wald e Wooldridge detectaram heterocedasticidade e autocorrelação, que foram corrigidos a partir dos erros-padrão clusterizados (Baum, 2006; Wooldridge, 2016).

No que tange ao melhor modelo para análise, foi aplicado o teste $\mathrm{F}$ de Chow, que rejeitou o modelo pooled e, posteriormente, o de Breusch-Pagan, que rejeitou a hipótese de modelo pooled a favor do modelo de efeitos aleatórios. Ao aplicar o teste de Hausmman, para sugerir qual seria o modelo mais apropriado, de efeitos fixos ou aleatório, rejeitou-se então o modelo de efeitos aleatórios, indicando que o modelo de efeitos fixos seria o mais adequado para estimar as regressões a partir das características dos dados coletados.

Para examinar a associação entre a política de dividendos e a VPA, a construção das variáveis e suas respectivas equações neste estudo, utiliza-se como base as pesquisas de Hashemijoo, Ardekani e Younesi (2012), Lashgari1 e Ahmadi (2014), Hooi, Albaity e Ibrahimy (2015) e Haque, Jahiruddin e Mishu (2019).

A variável dependente desta pesquisa é a volatilidade do preço das ações (VPA). Como variáveis independentes, este estudo empregou as principais variáveis da política de dividendos, sendo dividend yield e o índice de payout, conforme Zainudin, Mahdzan e Yet (2018). Tendo em vista que a volatilidade das ações pode ser influenciada pelo dividend yield e pelo payout, foram incluídas algumas variáveis de controle, de modo a garantir a robustez da análise. Assim, a Tabela 1 retrata as variáveis da amostra e suas equações, todas baseadas em teorias que reforçam a associação entre as variáveis utilizadas no modelo. 
Tabela 1. Descrição das Variáveis para a Pesquisa.

\begin{tabular}{|c|c|c|c|c|}
\hline Código & Equação & Descrição & Referencial $^{*}$ & SE \\
\hline \multicolumn{5}{|c|}{ Variável Dependente } \\
\hline$V P A$ & $V \cdot P A_{j}=\sqrt{\sum \frac{\left(S_{i}-S_{m}\right)^{2}}{n-1}}$ & $\begin{array}{l}\text { VPA = Vol. do Preço } \\
\text { das ações } \\
\mathbf{S}_{\mathbf{i}}=\text { Retorno } \\
\text { contínuo mensal da } \\
\text { ação i } \\
\mathrm{S}_{\mathrm{m}}=\text { média dos } \\
\text { retornos contínuos } \\
\text { mensais da ação i; } \\
\mathbf{I}=\text { igual a } 12,1 \text { ano }\end{array}$ & $\begin{array}{l}(1)(3)(4)(5) \\
(6)(7) \text { e }(8)\end{array}$ & \\
\hline \multicolumn{5}{|c|}{ Variáveis Independentes } \\
\hline D. yield & D. yield $=\frac{\text { Dividendos Pagos por Açẫo }}{\text { Valor Unitário }} \times 100$ & $\begin{array}{l}\text { D. } \text { yield }=\text { Rendimentos } \\
\text { dos Dividendos }\end{array}$ & $\begin{array}{l}(1)(2)(3)(4) \\
(5)(6)(7) \mathrm{e} \\
(8)\end{array}$ & $+/-$ \\
\hline Payout & Payout $=\frac{\text { Pagamento de DIvidendos }}{\text { Lucro Líquido }}$ & $\begin{array}{l}\text { Payout }=\text { Pagamento } \\
\text { de Dividendos }\end{array}$ & $\begin{array}{l}(1)(2)(3)(4) \\
(5)(6) \text { e }(7)\end{array}$ & $+/-$ \\
\hline & & $\begin{array}{l}\text { V.Luc }=\text { Volatilidade } \\
\text { dos Lucros }\end{array}$ & $\begin{array}{l}(3)(4)(5)(6) \\
(7) \text { e }(8)\end{array}$ & + \\
\hline V.Luc & 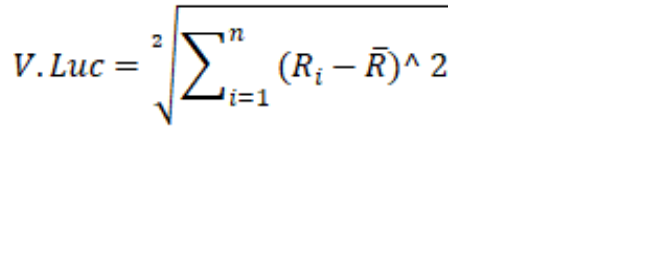 & $\begin{array}{l}\mathbf{R}_{\mathbf{i}}=\text { a razão entre a } \\
\text { receita operacional e o } \\
\text { ativo total do ano } \mathrm{i} \\
\bar{R}=\sum_{i=1}^{n} R_{i} / n \\
\mathbf{n}=(\text { de } 1 \text { a } 9) \text { indica } \\
\text { anos de } 2010 \text { a } 2018 .\end{array}$ & & \\
\hline V. Merc & $\begin{array}{l}\text { (* (Cotação x Total de Ações + Debêntures } \\
\text { CP (Curto Prazo) e LP (Longo Prazo) + } \\
\text { Financiamentos CP e LP + Adiantamentos de } \\
\text { Contratos de Câmbio - Disponibilidades e } \\
\text { Investimentos de Curto Prazo) / AT }\end{array}$ & $\begin{array}{l}\text { V. } \text { Merc = Valor de } \\
\text { Mercado }\end{array}$ & (2) & - \\
\hline Alav & Dívida Total/Ativo Total & Alav = Alavancagem & $\begin{array}{l}(3)(4)(5)(6) \\
\text { e (7) }\end{array}$ & $+/-$ \\
\hline Tam & Log Ativo Total & $\begin{array}{l}\text { Tam = Tamanho da } \\
\text { Empresa }\end{array}$ & $(5)$ & - \\
\hline
\end{tabular}

Nota: Variáveis: VPA: Volatilidade do preço da ação; D.yield: Divend Yield; Payout: Dividend Payout; V.Luc: Volatilidade do lucro; V.Mer: Valor de mercado; Alav: Alavancagem; Tam: Tamanho; SE: Sinal Esperado. *Descrição de Autores: (1) Baskin (1989), (2) Perobelli, Zanini e Santos (2009), (3) Hussainey, Mgbame e Chijoke-Mgbame (2011), (4) Hashemijoo, Ardekani e Younesi (2012), (5) Lashgaril e Ahmadi (2014) (6) Hooi, Albaity e Ibrahimy (2015), (7) Zainudin, Mahdzan e Yet (2018) e (8) Haque, Jahiruddin e Mishu (2019). Fonte: Autores.

Pela análise de estudos anteriores já mencionados, verificou-se que essas variáveis podem exercer influência na oscilação dos preços frente à distribuição de dividendos. Ademais, pretende-se por meio das Equações 1 e 2 avaliar tais influências:

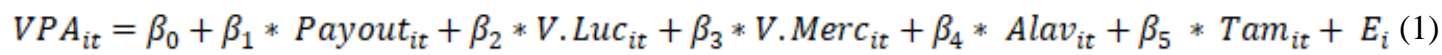

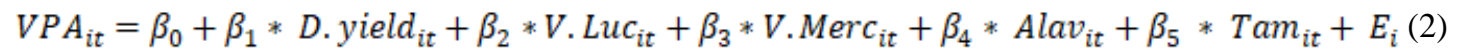

Onde,

$V \cdot P A_{i t}=$ Volatilidade do preço das ações da empresa i no tempo $t$

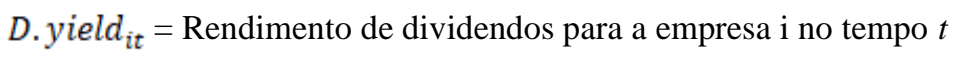

Payout $_{i t}=$ Taxa de pagamento da empresa i no tempo $t$ 
$V \cdot \operatorname{Merc}_{i t}=$ Valor de mercado da empresa i no tempo $t$

$V . L u c_{i t}=$ Volatilidade dos lucros da empresa i no tempo $t$

$\operatorname{Alav}_{i t}=$ Alavancagem é adicionada principalmente à equação de regressão como um controle variável i no tempo $t$

$\operatorname{Tam}_{i t}=$ Crescimento do ativo total para a empresa i no tempo $t$

$E_{i}=$ erro

A partir dos modelos formulados, propõem-se as hipóteses a seguir:

Hipótese 1: O Payout tem relação positiva com a volatilidade do preço das ações (Modelo 1)

Hipótese 2: O Rendimento dos Dividendos e a volatilidade do Lucro tem relação positiva com a Volatilidade do Preço das Ações (Modelo 2).

\section{Resultados e Discussão}

Os resultados da Tabela 2 apresentam os dados tratados após exclusão dos outliers, onde a variável dependente do estudo (VPA) apresentou um total de 1.380 observações, com uma média de aproximadamente 43\%, destacando-se a amplitude entre valores máximo e mínimo da variável.

Tabela 2. Estatística descritiva das variáveis.

\begin{tabular}{llllll}
\hline Variável & Observações & Média & Desvio Padrão & Mínimo & Máximo \\
\hline VPA & 1.380 & 42,997 & 21,078 & 15,447 & 197,975 \\
D.yield & 2.092 & 3,058 & 5,697 & 0 & 115,850 \\
Payout & 2.597 & 0,150 & 6,357 & 0 & 323,989 \\
V.Luc & 2.754 & 0,809 & 16,534 & 0,00002 & 772,379 \\
V.Merc & 2.202 & 0,816 & 1,076 & 0,0025 & 13,337 \\
Alav & 2.754 & 1,126 & 28,119 & 0 & $1.455,912$ \\
Tam & 2.754 & 14,549 & 2,127 & 3,239 & 20,769 \\
\hline
\end{tabular}

Nota: Variáveis: V.PA: Volatilidade do preço da ação; D.yield: Divend Yield; Payout: Dividend Payout; V.Luc: Volatilidade do lucro; V. Mer: Valor de mercado; Alav: Alavancagem; Tam: Tamanho. Fonte: Autores.

Já em relação às variáveis de interesse, o D.yield apresentou um total de 2.092, com uma média de aproximadamente R\$ 3.058/ação e o Payout 2,597 observações. Nesse sentido, com base no valor mínimo dessas variáveis, é possível observar que existem empresas que não distribuem dividendos como forma de remuneração ao acionista.

As variáveis V.Luc, Alav e Tam foram as que apresentaram o maior número de observações na amostra, com um total de 2.754. A discrepância entre os valores máximo e mínimo da variável tamanho, também reflete a diversidade de empresas componentes da amostra. A Tabela 3 apresenta a matriz de correlação de Person entre variáveis estudadas. A variável D.Yield apresentou uma relação negativa pequena, mas com relação definida, significativa ao nível de 5\% com a volatilidade do preço das ações (VPA).

A variável Payout também apresentou uma relação negativa pequena com variável dependente, porém, com uma intensidade maior quando comparada com o D.Yield. A relação se mostrou significante para uma alfa de 5\%. As variáveis Volatilidade do lucro (V.Luc) e Alavancagem (ALAV) apresentaram uma relação positiva e fraca com a VPA, ambas significativas ao nível de 5\%. Já as variáveis Valor de mercado (V.Merc) e tamanho (Tam) apresentaram uma relação negativa pequena com a VPA, significante a 5\%. Embora a V.Luc e o Payout possuam uma relação positiva muito forte, o teste VIF não indicou presença de multicolinearidade entre essas variáveis. Conforme a Tabela 4, o primeiro modelo destaca o uso apenas de pagamento de dividendos e o segundo somente rendimento de dividendos, seguidos das variáveis de controle. 
Tabela 3. Matriz de correlação entre as variáveis.

\begin{tabular}{llllllll}
\hline Variável & V.PA & D. yield & Payout & V.Luc & V.Merc & Alav & Tam \\
\hline VPA & 1,000 & & & & & & \\
D.yield & $-0,254^{*}$ & 1,000 & & & & & \\
Payout & $-0,299^{*}$ & $0,587^{*}$ & 1,000 & & & & \\
V.Luc & $0,195^{*}$ & $-0,037$ & $0,939^{*}$ & 1,000 & & & \\
V.Merc & $-0,210^{*}$ & 0,011 & $0,421^{*}$ & 0,041 & 1,000 & & \\
Alav & $0,254^{*}$ & $-0,042$ & $-0,003$ & 0,009 & $0,199^{*}$ & 1,000 & \\
Tam & $-0,338^{*}$ & $0,080^{*}$ & $-0,082^{*}$ & $-0,177^{*}$ & $-0,164^{*}$ & $-0,107^{*}$ & 1,000 \\
\hline
\end{tabular}

Nota: * indica significância a 5\%. Variáveis: V.PA: Volatilidade do preço da ação; D.yield: Divend Yield; Payout: Dividend Payout; V.Luc: Volatilidade do lucro; V.Mer: Valor de mercado; Alav: Alavancagem; Tam: Tamanho. Fonte: Autores.

Tabela 4. Regressão com Dados em Painel.

\begin{tabular}{|c|c|c|c|}
\hline & \multicolumn{2}{|c|}{ (Modelo 1) } & (Modelo 2) \\
\hline & $\stackrel{\mathrm{t}}{\operatorname{Std}{ }_{\mathrm{Frr}}}$ & & $(-2.80)$ \\
\hline Payout & $\stackrel{\mathrm{t}}{\mathrm{Std} . \mathrm{Err}}$ & $\begin{array}{c}-30.8092 * * \\
(-2.56) \\
(12.0246)\end{array}$ & \\
\hline V.Luc & $\begin{array}{c}\mathrm{t} \\
\text { Std. Err }\end{array}$ & $\begin{array}{c}-1.2777 \\
(-0.51) \\
(2.4832)\end{array}$ & $\begin{array}{c}-0.7252 \\
(-0.27) \\
(2.7138)\end{array}$ \\
\hline V.Merc & $\begin{array}{c}\mathrm{t} \\
\text { Std. Err }\end{array}$ & $\begin{array}{c}-2.6732 * * * \\
(-2.64) \\
(1.0110)\end{array}$ & $\begin{array}{c}-2.0416^{* *} \\
(-2.02) \\
(1.0129)\end{array}$ \\
\hline Alav & $\begin{array}{c}\mathrm{t} \\
\text { Std. Err }\end{array}$ & $\begin{array}{c}10.6009 \\
(1.45) \\
(7.2875)\end{array}$ & $\begin{array}{c}15.7232 * * * \\
(3.47) \\
(4.5324)\end{array}$ \\
\hline _cons & $\begin{array}{c}\mathrm{t} \\
\text { Std. Err }\end{array}$ & $\begin{array}{c}-5.4542 * * \\
(-2.23) \\
(2.4432) \\
121.6681^{* * *}\end{array}$ & $\begin{array}{c}-5.2181 * * \\
(-2.11) \\
(2.4712) \\
115.7259^{* * *}\end{array}$ \\
\hline $\mathrm{N}$ & & 1341 & 1369 \\
\hline R-sq & & 0.239 & 0.246 \\
\hline adj. R-sq & & 0.231 & 0.239 \\
\hline rmse & & 10.9344 & 11.3304 \\
\hline $\begin{array}{c}\text { VIF } \\
\text { Hausman }\end{array}$ & & $\begin{array}{c}1,32 \\
0.0017\end{array}$ & $\begin{array}{c}1,08 \\
0.0000\end{array}$ \\
\hline
\end{tabular}

Nota: *. **. *** indicam nível de significância a 10\%, 5\% e 1\% respetivamente. Variáveis: V.PA: Volatilidade do preço da ação; D.yield: Divend Yield; Payout: Dividend Payout; V.Luc: Volatilidade do lucro; V.Mer: Valor de mercado; Alav: Alavancagem; Tam: Tamanho. Std. Err: Erro Padrão; t: Teste T. Os testes Breusch-Pagan LM, Chow, Wooldridge e Wald apresentaram uma significância de 0,0000 para ambos os modelos. Fonte: Autores.

Assim, de acordo com o Modelo 1, a política de dividendos adotada pela empresa exerce influência sobre a volatilidade do preço da ação da mesma, de forma negativa e com nível de significância de 5\%, indicando que para cada unidade aumentada da variável Payout, a VPA tenderá a diminuir aproximadamente 30,8 unidades. Esse resultado contradiz os encontrados por Hashemijoo, Ardekani e Younesi (2012) e Silva Júnior e Machado (2015), que não encontram relação entre as duas variáveis, mas está de acordo com Lashgaril e Ahmadi (2014) e Hooi, Albaity e Ibrahimy (2015).

No Modelo 2, a variável $D$. yield apresentou uma relação negativa com a volatilidade do preço das ações. A relação é estatisticamente significativa ao nível de $1 \%$ e indica que a cada unidade a mais de D.yield da empresa, a volatilidade do preço da ação tenderá a diminuir em aproximadamente 0,24 unidades. As duas variáveis relacionadas à política de dividendos apresentaram relação negativa com a volatilidade do preço. O resultado está convergente com as pesquisas realizadas por Hashemijoo, Ardekani e Younesi (2012), Hooi, Albaity e Ibrahimy (2015) e Silva Júnior e Machado (2015). 
O resultado da relação negativa entre a política de dividendos (representada pelo D.yield e Payout) pode ser explicado pelo interesse dos investidores em aplicar seus recursos em empresas que distribuem mais dividendos. Além disso, uma distribuição de um maior montante de dividendos transmite ao mercado que a empresa está tendo lucros, com boas perspectivas para o futuro da organização. Dessa forma, a distribuição de dividendos se mostra como uma forma de atrair investidores para a companhia (Baskin, 1989; Hool, Albaity \& Ibrahumy, 2015).

Nos modelos analisados, a variável volatilidade dos lucros (V.Luc) não apresentou significância estatística, de modo que não se pode inferir que algum tipo de influência seja positiva ou negativa sobre a volatilidade do preço das ações das empresas brasileiras de capital aberto. Assim, a presente pesquisa não confirma os estudos de Baskin (1989), Hashemijoo, Ardekani e Younesi (2012) e Hooi, Albaity e Ibrahimy (2015), que apontam que uma perspectiva positiva de lucro ao longo do tempo tendem a atrair mais investidores.

O valor de mercado da companhia (V.Mer) também apresentou significância estatística ao nível 1\% para o primeiro modelo analisado e 5\% para o segundo modelo. Essa variável influencia de forma negativa a volatilidade do preço das ações, pois empresas que possuem um maior valor de mercado tendem a ter um maior acesso ao mercado de capitais e maior facilidade de captar recursos para realizar novos investimentos (Alzomaia \& Al-Khadhiri, 2013; Hool, Albaity \& Ibrahumy, 2015).

Em relação à Alavancagem (Alav), a variável não apresentou significância estatística no modelo 1, porém, no Modelo 2, a variável foi estatisticamente significante para um alfa de $1 \%$. A alavancagem influencia de forma positiva a volatilidade do preço das empresas, uma vez que empresas mais endividadas tenderão a diminuir as expectativas dos investidores. Baskin (1989), Hooi, Albaity e Ibrahimy (2015) encontraram resultados semelhantes ao desta pesquisa, entretanto Hashemijoo, Ardekani e Younesi (2012) não encontraram relação entre ambas as variáveis.

Já o tamanho exerce uma relação negativa na volatidade do preço das ações das empresas brasileiras, tal relação é estatisticamente significante ao nível de 5\% para os dois modelos. Essa relação ocorre porque empresas que possuem uma maior diversificação de seus produtos e uma operação mais consolidada leva a uma maior estabilidade do preço de suas ações. Já as empresas menores tendem a ser mais instáveis quanto à volatilidade de suas ações (Baskin, 1989). Autores como Hashemijoo, Ardekani e Younesi (2012), Hooi, Albaity e Ibrahimy (2015) confirmaram essa relação em suas pesquisas, entretanto, Lashgaril e Ahmadi (2014) não encontraram significância estatística nessa relação.

De acordo com os resultados da pesquisa, não se pode inferir que o pagamento de dividendos aumenta a volatilidade do preço das ações, não confirmando a primeira hipótese deste estudo, assim como, a não confirmação do rendimento dos dividendos com a volatilidade do preço das ações para a segunda hipótese do estudo, porém a variável de volatilidade do lucro não apresentou significância estatística para se inferir sobre sua influência na volatilidade das ações.

\section{Conclusão}

Esta pesquisa investigou a relação entre distribuição de dividendos e a volatilidade das ações de empresas brasileiras listadas na B3 no período de 2010 a 2018. Para mensurar a relação entre as variáveis foi realizado dois modelos a fim de identificar as implicações das principais variáveis da política de dividendos (dividend yield e payout).

O procedimento metodológico envolveu técnicas de regressão linear múltipla com dados em painel de efeitos fixos. Os modelos apresentam a associação da política de dividendos - dividend yield e payout - em relação à volatilidade do preço da ação (VPA). O primeiro modelo destaca o uso apenas de pagamento de dividendos e o segundo modelo somente rendimento de dividendos, seguidos das variáveis de controle.

Os resultados da pesquisa, utilizando dois modelos de regressões (modelo 1 e 2), apontaram que as principais variáveis para a política de dividendos apresentaram significância em relação à volatilidade do preço das ações. Não obstante, 
a variável de volatilidade dos lucros, que é uma medida usada pelas empresas para distribuir os lucros de volta aos acionistas e que indiretamente possui relação com a política de dividendos das empresas, não se mostrou significante nos modelos, de maneira que não se pode inferir sobre a necessidade das relações entre a política de lucros das empresas e a volatilidade do preço das ações.

Os modelos apresentaram uma relação negativa e significante com a volatilidade do preço das ações, de modo que, se um aumenta o outro diminui, isto é, dado uma redução no dividend yield e no pagamento de dividendos há um aumento na volatilidade do preço das ações das empresas brasileiras. Desta forma, os resultados comprovam o que se esperava neste estudo: a política de dividendos influencia negativamente a volatilidade dos preços das ações. Além disso, é importante salientar que segundo Hooi, Albaity e Ibrahimy (2015) o pagamento e o rendimento de dividendo estão entre os principais fatores que os investidores julgam ao analisar uma decisão de investimento.

Esta pesquisa vai ao encontro dos achados de Haque, Jahiruddin e Mishu (2019), apresentando uma relação negativa entre valor de mercado e volatilidade do preço das ações, corroborando que empresas que retêm maior valor de mercado possuem menor dependência da relação com a VPA, pois dispõem de melhor acesso a recursos e fundos para investimentos e pagamentos.

Em relação à alavancagem no segundo modelo, a variável se mostrou positiva e significativa com a volatilidade dos preços, sugerindo que quanto mais alavancada for uma empresa, mais volátil será o preço das ações. Argumenta-se que empresas maiores sejam menos arriscadas e tenham menor volatilidade do preço das ações, pois são possivelmente mais diversificadas e possuem maior acesso a recursos financeiros.

No que se refere às hipóteses deste estudo, os resultados não comprovaram que o aumento no pagamento de dividendos aumenta a volatilidade do preço das ações, revelando um seguimento contrário à primeira hipótese, assim como a não confirmação do rendimento dos dividendos com a volatilidade do preço das ações para a segunda hipótese do estudo. Em relação à influência da volatilidade do lucro, não se pode inferir sobre sua influência na volatilidade das ações.

Em síntese, as descobertas desta pesquisa trouxeram implicações práticas para investidores e para empresas de capital aberto que utilizam de diferentes estratégias, pois através das informações sobre o dividend yield e o pagamento de dividendos, ou seja, através da política dos lucros os investidores podem analisar e executar uma estratégia mais precisa sobre a análise financeira da empresa e de seus investimentos.

Como limitação, a presente pesquisa não distinguiu os juros sobre capital próprio do pagamento de dividendos, tendo em vista que embora ambos sejam formas de remuneração dos acionistas, possuem suas diferenças. Para pesquisas futuras, sugere-se a comparação entre essas duas formas de remuneração, a utilização de outras técnicas estatísticas e até mesmo uma comparação com outros países.

\section{Referências}

Al-Malkawi, Husam Aldin N. (2007). Determinants of corporate dividend policy in Jordan: an application of the Tobit model. Journal of Economic and Administrative Sciences, 23(2), 44-70.

Alzomaia, T. S. F. \& Al-Khadhiri, A. (2013). Determination of dividend policy: The evidence from Saudi Arabia. International Journal of Business and Social Science, 4(1).

Ball, R. et al. (1979). Dividends and the value of the firm: evidence from the Australian equity market. Australian Journal of Management, 4(1), 13-26.

Barberis, N., Huang, M. \& Santos, T. (2001). Prospect theory and asset prices. The quarterly journal of economics, 116(1), 1-53.

Baskin, J. (1989). Dividend policy and the volatility of common stocks. Journal of portfolio Management, $15(3), 19$.

Baum, C. F. (2006). An introduction to modern econometrics using Stata. Texas: Stata press.

Black, F. \& Scholes, M. (1974). The effects of dividend yield and dividend policy on common stock prices and returns. Journal of financial economics, 1(1), $1-22$. 
Elton, E. J. \& Gruber, M. J. (1970). Marginal stockholder tax rates and the clientele effect. The Review of Economics and Statistics, 68-74.

Fama, E. F. et al. (1969). The adjustment of stock prices to new information. International economic review, 10(1), 1-21.

Fávero, L. P. et al. (2009). Análise de dados: modelagem multivariada para tomada de decisões. Elsevier.

Forti, C. A. B., Peixoto, F. M. \& Alves, D. L. (2015). Fatores determinantes do pagamento de dividendos no Brasil. Revista Contabilidade \& Finanças, 26(68), 167-180.

Gordon, M. J. (1963). Optimal investment and financing policy. The Journal of finance, 18(2), $264-272$.

Haque, R., Jahiruddin, A. T. M. \& Mishu, F. (2019). Dividend Policy and Share Price Volatility: A Study on Dhaka Stock Exchange. Australian Academy of Accounting and Finance Review, 4(3), 89-99.

Hashemijoo, M., Ardekani, A. M. \& Younesi, N. (2012). The impact of dividend policy on share price volatility in the Malaysian stock market. Journal of business studies quarterly, 4(1).

Hooi, S. E., Albaity, M. \& Ibrahimy, A. I. (2015). Dividend policy and share price volatility. Investment Management and Financial Innovations, 12(1), 226234.

Hussainey, K., Mgbame, C. O. \& Chijoke-Mgbame, A. M. (2011). Dividend policy and share price volatility: UK evidence. The Journal of risk finance, 12(1), 57-68.

Khan, F., Jan, SU e Khan, H. (2020). A política de dividendos determina a volatilidade do preço das ações? (Um estudo de caso do setor manufatureiro da Malásia). Journal Global Policy and Governance, 9 (1), 67-78.

Lashgari, Z. \& Ahmadi, M. (2014). The impact of dividend policy on stock price volatility in the Tehran stock exchange. Kuwait Chapter of the Arabian Journal of Business and Management Review, 3(10), 273.

Leite, M., Bambino, A. C. \& Hein, N. (2017). Relação entre política de dividendos e desempenho econômico financeiro em empresas brasileiras e chilenas. Revista de Gestão, Finanças e Contabilidade, 7(1), 205-221.

Leroy, S. F. \& Porter, R. D. (1981). The present-value relation: Tests based on implied variance bounds. Econometrica: Journal of the Econometric Society, $555-574$.

Lintner, J. (1956). The distribution of incomes of corporations among dividends, retained earnings and taxes. The American Economic Review, v. 46.

Loss, L. \& Sarlo Neto, A. (2003). Política de dividendos, na prática, é importante? Revista Contabilidade \& Finanças, 14, 39-53.

Martins, A. I. \& Famá, R. (2012). O que revelam os estudos realizados no Brasil sobre política de dividendos? Rae-Revista De Administracao De Empresas, 52(1), 24-39.

Miller, M. \& Modigliani, F. (1961). Dividend policy, growth, and the valuation of shares. The Journal of Business, 34(4), 411-433.

Medeiros, O. R. \& Van Doornik, B. F. N. (2008). A relação empírica entre dividendos, volatilidade de retornos e volume de negócios no mercado de ações brasileiro. BBR-Brazilian Business Review, 5(1), 1-17.

Pietro Neto, J., Decourt, R. F. \& Galli, O. (2011). C. Proventos-A teoria da sinalização: variações de mercado. Revista de Administração FACES Journal, $10(4), 150-168$

Phan, T. K. H. \& Tran, N. H. (2019). Política de dividendos e volatilidade do preço das ações em um mercado emergente: a estrutura de propriedade importa?. Cogent Economics \& Finance, 7 (1), 1637051. 10.1080 / 23322039.2019.1637051

Perobelli, F. F. C., Zanini, A. \& Santos, A. B. (2009). Pagamento de proventos versus preços deações maduras e em expansão segundo Kohonen Maps. Revista de Administração de Empresas, 49(2), 132-146.

Pettit, R. R. (1972). Dividend announcements, security performance, and capital market efficiency. The Journal of Finance, $27(5), 993-1007$.

Procianoy, J. L. \& Poli, B. T. C. (1993). A política de dividendos como geradora de economia fiscal e do desenvolvimento do mercado de capitais: uma proposta criativa. Revista de Administração de Empresas, 33(4), 06-15.

Procianoy, J. L \& Verdi, R. S. (2009). Clientela em dividendos, novos elementos e novas questões: o caso brasileiro. RAE- eletrônica, 8(1).

Procianoy, J. L \& Verdi, R. S. (2003). O efeito clientela no mercado brasileiro: será que os investidores são irracionais? Revista Brasileira de Finanças, 1(2), $217-242$.

Rodrigues, D. R. et al. (2019). Determinantes Da Distribuição De Dividendos No Brasil: Uma Análise Entre Flexibilidade Financeira E Política De Distribuição De Resultados. Contabilidade Vista \& Revista, 30(2), 84-112.

Santos Marques, B., Perpétuo, A. A. G., \& Rocha, D. M. (2020). Análise bibliométrica sobre os estudos de dividendos no Brasil. Research, Society and Development, 9(8), e463985658-e463985658.

Shiller, R. J. (2003). From efficient markets theory to behavioral finance. Journal of economic perspectives, 17(1), 83-104.

Silva, C. P. \& Machado, M. A. (2015). A Influência Da Política De Dividendos Sobre A Volatilidade Das Ações. Revista Contabilidade e Controladoria, 7(3).

Sousa, T. F. et al. (2018). Estudo bibliométrico sobre dividendos no Brasil. Capital Científico, 16(3). 
Research, Society and Development, v. 10, n. 4, e42910414210, 2021

(CC BY 4.0) | ISSN 2525-3409 | DOI: http://dx.doi.org/10.33448/rsd-v10i4.14210

Wooldridge, J. M. (2016). Introductory econometrics: A modern approach. Nashville: Nelson Education.

Zainudin, R., Mahdzan, N. S. \& Yet, C. H. (2018). Dividend policy and stock price volatility of industrial products firms in Malaysia. International Journal of Emerging Markets, 13(1), 203-217. 